\title{
Thymoquinone prevents $\beta$-amyloid neurotoxicity in primary cultured cerebellar granule neurons
}

\begin{abstract}
Thymoquinone (TQ), a bioactive constituent of Nigella sativa Linn (N. sativa) has demonstrated several neuropharmacological attributes. In the present study, the neuroprotective properties of TQ were investigated by studying its anti-apoptotic potential to diminish $\beta$-amyloid peptide $1-40$ sequence (A $\beta 1-40)$-induced neuronal cell death in primary cultured cerebellar granule neurons (CGNs). The effects of TQ against A $\beta 1-40$-induced neurotoxicity, morphological damages, DNA condensation, the generation of reactive oxygen species, and caspase-3, -8, and -9 activation were investigated. Pretreatment of CGNs with TQ $(0.1$ and $1 \mu \mathrm{M})$ and subsequent exposure to $10 \mu \mathrm{M} \mathrm{A} \beta 1-40$ protected the CGNs against the neurotoxic effects of the latter. In addition, the CGNs were better preserved with intact cell bodies, extensive neurite networks, a loss of condensed chromatin and less free radical generation than those exposed to A $\beta 1-40$ alone. TQ pretreatment inhibited $A \beta 1-40$-induced apoptosis of CGNs via both extrinsic and intrinsic caspase pathways. Thus, the findings of this study suggest that TQ may prevent neurotoxicity and A $\beta 1-40$-induced apoptosis. TQ is, therefore, worth studying further for its potential to reduce the risks of developing Alzheimer's disease.
\end{abstract}

Keyword: Thymoquinone; $\beta$-Amyloid; Alzheimer`s disease; Neurotoxicity; Primary cultured cerebellar granule neurons 